ISSN 1112-9867

Available online at

http://www.jfas.info

\title{
GAP IN A LASER FREQUENCY He-Ne AT 632.8 nm
}

\author{
A. Aissani ${ }^{*}$, M. Alliche, M. Chaalal and O. Ziane. \\ Laboratoire d'Electronique Quantique - Faculté de Physique- USTHB
}

Received: 25 November 2012 / Accepted: 02 December 2012 / Published online: 30 June 2012

\begin{abstract}
The aim of this work is to show that the emitted signals of a double-output laser cavity exhibit distinctive features, manifesting themselves through typical dissymmetry in the corresponding spectral line shapes. Usually, such a dissymmetry in an inhomogeneously broadened gas laser appears with a clear but unpredictable shift of the maximum intensity level either towards the high or towards the low frequency sides with respect to line centre. In the case of a double-output laser, the spectral profiles show opposite shifts. This means that when the maximum intensity of one output moves towards the high frequency side of the profile, the maximum intensity of the other moves towards the low frequency side. This gives rise to a frequency shift, with respect to the Lamb-dip for a classical laser

On the theoretical side, we apply the standard disturbed Gaussian beam model to give a good quantification of the frequency shifts obtained at both sides of the system.
\end{abstract}

Key words: spectral line, double-output laser, frequency shift.

\section{INTRODUCTION}

La raie d'émission d'un laser à gaz à faible gain à une sortie est caractérisée par un Lamb-dip généralement dissymétrique [1-2]. Plusieurs causes sont à l'origine de cette dissymétrie [3]. Ces causes concourent pour créer des inhomogénéités de population et de saturation, qui induisent des effets lentilles et modifient le rayon de courbure du front d'onde et le diamètre du mode [4].

Author Correspondence, e-mail: aaissani@yahoo.fr ICID: 1020745 
Pour un tel laser, les effets d'hétérogénéité de population sont opposés à ceux de la saturation; les premiers donnent une forme de raie dissymétrique du coté des hautes fréquences, tandis que les seconds donnent un maximum du coté des basses fréquences. D'autres études [5-6] ont montré que les paramètres géométriques de la cavité influent sur la forme de raie. En effet, ceux-ci modulent la compétition entre les deux types d'hétérogénéité et peuvent donc favoriser l'un ou l'autre.

En métrologie cette dissymétrie de la raie est indésirable pour l'établissement d'un standard de fréquence [7-8]. Pour réaliser alors un étalon fiable de fréquences, il est nécessaire de situer la fréquence du creux du Lamb-dip par rapport à la fréquence centrale de résonance.

Le but de ce travail est de montrer que l'intensité d'un laser fonctionnant en double sortie [9] n'est pas la même aux deux extrémités de la cavité. Il en découle une différence de dissymétrie dans la courbe de gain induisant une dérive de la fréquence du creux du Lamb-dip par rapport à la fréquence de résonance du laser. En utilisant le modèle du faisceau Gaussien perturbé, nous montrons qu'il est possible de mesurer ce décalage en fréquence entre les signaux de sortie aux deux extrémités.

\section{MODELE THEORIQUE}

L'expression des composantes aller (indicée «f») et retour (indicée «b ») du champ résonant dans la cavité de la figure1est donnée par [10]:

$$
E_{f, b}(\rho, \zeta)=\frac{E_{0 f, b}}{1 \pm 2 i \zeta} \exp \left[ \pm i\left(k \frac{\omega_{o}}{c} W_{o}^{2}\right) \zeta\right] \cdot \exp \left(-\frac{\rho^{2}}{1 \pm 2 i \zeta}\right) \cdot \exp \left[-i\left(\varepsilon_{f, b} \mp \frac{\rho^{2}}{2} \eta_{f, b}\right)\right]
$$

Où $E_{0 f, b}$ sont les amplitudes complexes du champ, $\varepsilon_{f, b}$ représente la perturbation du paramètre longitudinal (phase et amplitude) et $\eta_{f, b}$ celle du paramètre transversal (diamètre et rayon de courbure du mode), $2 W_{0}$ est le diamètre du mode au pincement, $\omega_{0}$ est la fréquence centrale, $c$ est la vitesse de la lumière dans le vide, $k$ est la constante de propagation. et $(\rho, \zeta)$ sont les coordonnées réduites reliées aux coordonnées cylindriques $(r, z)$ par: $\rho=r / w_{0} ; \zeta=c z / \omega_{0} w_{0}^{2}$. Le signe du haut dans le symbole « \pm » ou «₹» se rapporte à l'onde aller et celui du bas à l'onde retour. 
Compte tenu des conditions aux limites en $z_{1}$ et $z_{2}$ et des conditions de continuité en $z_{3}$ et $\mathrm{z}_{4}$, on peut exprimer la puissance totale du coté 1 en fonction de celle du coté 2 . On obtient [10]:

$$
\begin{aligned}
P\left(\zeta_{1}\right)= & P\left(\zeta_{2}\right) \cdot\left[\frac{2-\left(1+4 \zeta_{2}^{2}\right) \eta_{b_{2}}^{i}}{2-\left(1+4 \zeta_{1}^{2}\right) \eta_{b_{1}}^{i}}\right] \cdot\left[1-\exp \left(-\frac{b^{2}}{W_{0}^{2}} \cdot \frac{2-\left(1+4 \zeta_{1}^{2}\right) \eta_{b 1}^{i}}{\left(1+4 \zeta_{1}^{2}\right)}\right)\right] \\
& \exp \left[-2\left(\frac{\omega_{0}}{c} W_{0}^{2}\right) \cdot\left(k_{g}^{i} l_{g}\right)\right] \cdot \exp \left(2 \varepsilon_{B}^{i}\right)
\end{aligned}
$$

Où l'exposant «i » signifie la partie imaginaire de la grandeur considérée, $k_{g}^{i}$ est la constante de propagation dans le milieu actif, $l_{g}$ sa longueur réduite, $\eta_{b j}^{i}$ le paramètre transverse $\eta_{b}$ au point $z_{j}$ et $\varepsilon_{B}$ est donné par:

$$
\varepsilon_{B}=\left({ }^{1} \varepsilon_{b_{1}}-{ }^{1} \varepsilon_{b_{3}}\right)+\left({ }^{2} \varepsilon_{b_{3}}-{ }^{2} \varepsilon_{b_{4}}\right)+\left({ }^{3} \varepsilon_{b_{4}}-{ }^{3} \varepsilon_{b_{2}}\right) .
$$

On remarque, à travers la relation (2), que $P\left(\zeta_{1}\right)$ est différent de $P\left(\zeta_{2}\right)$, Ce qui explique l'obtention de formes de raie différentes aux deux sorties de la cavité. On se propose alors de quantifier, dans ce qui suit, ce déplacement en fréquence.

Rappelons que dans un laser à gaz à faible gain, la raie d'émission est caractérisée par un minimum dont la fréquence $\omega_{L D}$ est, en général, différente de la fréquence centrale $\omega_{0} \mathrm{du}$ laser. Il est donc intéressant de voir, par exemple, comment elle varie en fonction de la position $z_{3}$ du tube. Pour cela, la méthode utilisée consiste à faire au voisinage de la fréquence centrale, un développement limité au $2^{\text {ième }}$ ordre de l'expression de la puissance et ensuite de traduire le fait que le Lamb-dip, est caractérisé par un minimum d'intensité. La dérivée de cette dernière est alors nulle en ce point. Cette démarche permet d'obtenir la relation :

$$
x_{L D}=-\left[\frac{d P_{i}}{d x}\right]_{x=0} /\left[\frac{d^{2} P_{i}}{d x^{2}}\right]_{x=0}
$$

Où $x=\left(\omega-\omega_{0}\right) / \Gamma$ est la fréquence réduite et $\Gamma$ la demi largeur Doppler.

Cette relation permet de calculer l'écart entre les fréquences $\omega_{L D}$ et $\omega_{0}$ ce qui permet de donner la valeur de l'écart fréquentiel $\left(\omega_{L D 2}-\omega_{L D 1}\right)$ entre les deux sorties de la cavité. 


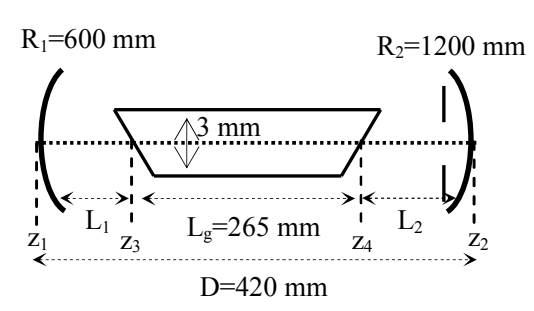

Fig.1. Cavité laser

\section{RÉSULTATS NUMÉRIQUES}

Le calcul numérique est effectué pour un laser He-Ne oscillant sur la raie $6328 \mathrm{~A}^{\circ}$. La cavité correspondante est celle de la Figure1. Les courbes obtenues (Fig.2) montrent une dissymétrie, de type HF, différente pour les deux sorties de la cavité. Elle est plus accentuée du coté2, ce qui est prévisible car le faisceau est plus large. Les variations du diamètre du mode oscillant (Figure 3) présente une forme de dispersion avec un maximum du coté $\mathrm{BF}$, ce qui génère des pertes plus grandes de ce côté. Ceci explique la dissymétrie de type HF en accord avec les figures a-b. En outre, les variations sont plus importantes du coté 2 du laser ce qui confirme la forte dissymétrie de ce côté là.
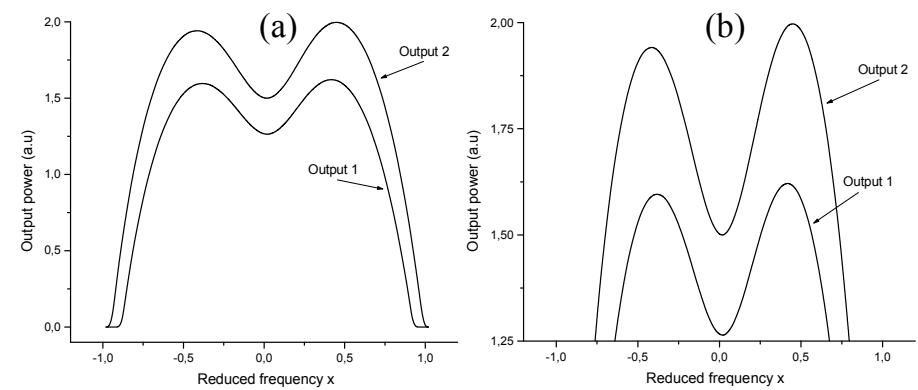

Fig.2. (a) formes de raie (puissance de sortie en fonction de la fréquence. (b) Zoom de (a)

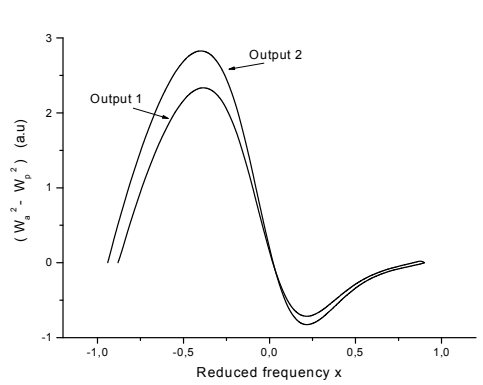

Fig.3. Variation en fonction de la fréquence de $\left(W^{2}-W_{0}^{2}\right)$

Quant aux courbes de la figure 4, elles donnent l'écart fréquentiel entre la fréquence centrale et celle du Lamb-dip en fonction de la position du tube amplificateur. Dans les deux cas, l'écart est systématiquement positif ce qui donne une dissymétrie de type HF et ce quel que soit la position du tube dans la cavité. Par contre, elle est plus grande du 
coté 2 pour les mêmes raisons citées précédemment. En outre, lorsque le tube se rapproche de la sortie 2 , donc s'éloigne du coté 1 , l'écart en fréquence diminue du coté 2 et augmente du coté1 ce qui est normal puisque le faisceau s'élargit à droite et se rétrécie à gauche. Les inhomogénéités de population augmentent donc du coté 2 et diminuent du coté 1 alors que pour celles de saturation, c'est l'inverse.

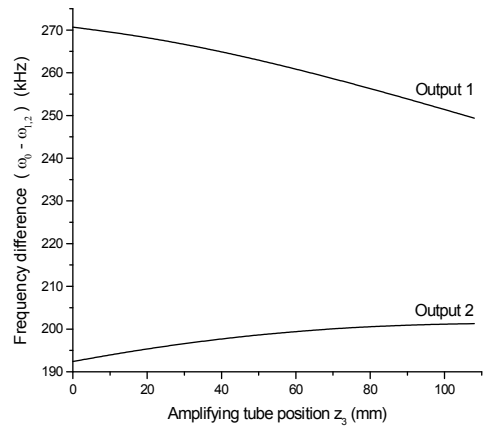

Fig.4. Variation en fonction de la position du tube amplificateur de

$$
\left(\omega_{0}-\omega_{L D 1,2}\right)
$$

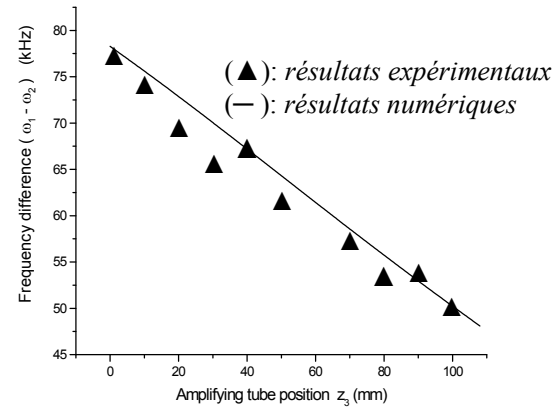

Fig.5. Variation en fonction de la position du tube amplificateur de

$$
\left(\omega_{L D 2}-\omega_{L D 1}\right)
$$

Enfin, la courbe de la figure 5, déduite de celles de la figure 4, représente l'écart fréquentiel entre les deux Lamb-dip aux deux extrémités du laser, en fonction de la position du milieu actif dans la cavité.

\section{ETUDE EXPÉRIMENTALE}

L'étude expérimental, a été faite sur une cavité correspondant de près à celle étudiée numériquement. La pression totale à l'intérieur du tube est de 0,6 torr. La figure 6 donne les formes de raie enregistrées sur un oscilloscope. Nous constatons une dissymétrie de type HF. Les inhomogénéités de population l'emportent donc sur celles de saturation et sont plus élevées du coté2 en accord avec le calcul numérique.

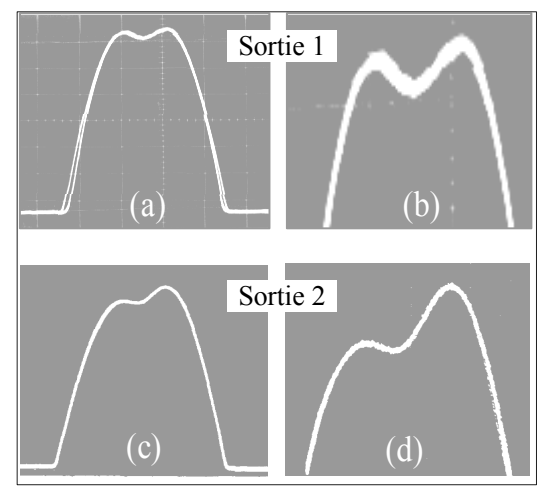

Fig.6. (a, c) Formes de raies). (b, d) Zoom sur le Lamb dip 
Les résultats de mesures du décalage en fréquence $\left(\omega_{L D 2}-\omega_{L D 1}\right)$ entre les deux sorties, sont données en figure 5. On constate alors un comportement similaire à celui obtenu numériquement.

\section{CONCLUSION}

Le modèle présenté ici montre qu'il existe une différence de dissymétrie entre les raies d'émission aux deux sorties du laser. Cette différence de dissymétrie induit un déplacement en fréquence d'un bout à l'autre du laser. L'intérêt de notre modèle est qu'il a permis d'expliquer cet effet et de le quantifier.

\section{REFERENCES}

[1] Lamb W. E. Theory of an Optical Maser. Jr. Phys. Rev. 1964, 134, A1429.

[2] Szoke A., Javan A. Isotope Shift and Saturation Behaviour of the 1.15- $\mu$ Transition of Ne. Phys. Rev. Lett. 1963, 10, 521.

[3] Szoke A., Javan A. Effects of Collisions on Saturation Behavior of the 1.15- $\mu$ Transition of Ne Studied with He-Ne Laser. Phys. Rev. 1966, 145, 137.

[4] Cordover R. H., Bouczyk P. A. Effects of Collisions on the Saturation Behavior of the $6328 \mathrm{~A}^{\circ}$ Transition of Ne Studied with a He-Ne Laser. Phys. Rev. 1969, 188, 696.

[5] Stephan G., Trumper H. Macroscopic parameters and line shapes of a gas laser. Phys. Rev. 1984, A30, 1925.

[6] Stephan G., Hugon D. Light Polarization of a Quasi Isotropic Laser with Optical Feedback. Phys. Rev. Lett. 1985, 55, 703.

[7] Le Floch A., Le Naour R., Lenormand J. M., Tache J. P. A critical geometry for lasers with internal lens like effects. J. Phys. Lett. 1980, 45, 544.

[8] Bartl J., Guttennova J., Jacko V., Sevcik R. Circuits for Optical Frequency Stabilization of Metrological Lasers. Measurement science review. 2007, 7, 59.

[9] Battou K., Ait-Ameur K., Ziane O. Q-switch of a continuously pumped CO2 laser with a scanning coupled-cavity Michelson mirror. Opt.Commun. 2008, 281, 5234.

[10] Aissani A., Meziane B., Battou K. et Ziane O. Spectral-Line Asymmetry in Double-Output-Cavity Laser. Optics \& Laser Technology. 2010, 42, 439-446. 


\section{ECART FREQUENTIEL DANS UN LASER He-Ne A 632.8 nm}

\section{RESUME}

Le but de ce travail est de montrer que l'intensité d'un laser fonctionnant en double sorties n'est pas la même aux deux extrémités de la cavité. En général, la forme de raie présente une dissymétrie qui se traduit par un maximum du côté des basses ou des hautes fréquences. Il en découle une différence de dissymétrie qui apparaît dans la courbe de gain de part et d'autre du laser induisant un déplacement, par rapport à la fréquence de résonance du laser, de la fréquence du creux du Lamb-dip, pour un laser classique ou celle du pic d'absorption saturé, pour un laser métrologique.

En utilisant le modèle usuel du faisceau Gaussien perturbé, nous montrons qu'il est possible de mesurer ce décalage en fréquence entre les signaux de sortie aux deux extrémités. Cette différence est associée à une dissimilitude dans la dissymétrie de la forme de raie qui se traduit par un déplacement du creux du Lamb-dip.

Mots-clés: forme de raie, laser à double sorties, déplacement en fréquence.

\section{How to cite this article}

Aissani A, Alliche M, Chaalal M and Ziane O. Frequency difference in a laser He-Ne A 632.8 nm. J Fundam Appl Sci. 2012, 4(1), 39-45. 